\title{
First results of a high precision mass measurement program for very short-lived nuclides
}

\author{
C. Toader ${ }^{\mathrm{a}, 1}$, C. Monsanglant ${ }^{\mathrm{a}}, \mathrm{G}$ G. Audi ${ }^{\mathrm{a}}$, G. Conreur $^{\mathrm{a}}$, \\ H. Doubre ${ }^{\text {a }}$, S. Henry ${ }^{\text {a }}$, M. Jacotin ${ }^{\text {a }}$, J.F. Kepinski ${ }^{\text {a }}$, \\ G. Le Scornet ${ }^{\mathrm{a}}$, D. Lunney ${ }^{\mathrm{a}}$, M. De Saint Simon ${ }^{\mathrm{a}}$, \\ C. Thibault ${ }^{a}$, H.-J. Kluge ${ }^{\mathrm{b}}$, C. Borcea ${ }^{\mathrm{c}}$, M. Duma ${ }^{\mathrm{c}}$ \\ and G. Bollen ${ }^{\mathrm{d}}$ \\ ${ }^{a}$ CSNSM, Bat 108, F-91405 Orsay \\ ${ }^{\mathrm{b}}$ GSI, Planckstrasse 1, D-64291 Darmstadt \\ ${ }^{\mathrm{c}}$ INPE, P.O. Box MG-6, RO-76900 Bucharest-Magurele

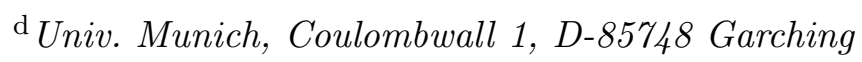 \\ ${ }^{\mathrm{e}}$ and the ISOLDE collaboration CERN, CH-1211 Geneva 23
}

\begin{abstract}
The recent commissioning of the experimental program MISTRAL (Mass Measurements at ISolde using a Transmission and Radiofrequency spectrometer on Line) brought mass measurements of high precision performed on sodium and magnesium isotopes. The shortest half-life of an isotope measured with MISTRAL was $30.5 \mathrm{~ms}$ $\left({ }^{28} \mathrm{Na}\right)$. Though this first period of data taking was dedicated to the exploration of MISTRAL performances it has allowed us to reduce the uncertainty in the mass of the ${ }^{28-30} \mathrm{Na}$ isotope by almost one order of magnitude.
\end{abstract}

Key words: mass spectrometry; short-lived nuclides

Verifying binding energies and minimizing their uncertainties in the neutron rich sodium region of the nuclear chart is important to clarify the long standing problem of the strength of the $\mathrm{N}=20$ shell closure.

The new experimental program MISTRAL (Mass Measurements at ISolde using a Transmission and Radiofrequency spectrometer on Line) was installed

$\overline{1 \text { Now }}$ at GSI Darmstadt

Preprint submitted to Elsevier Preprint 19 July 2000 


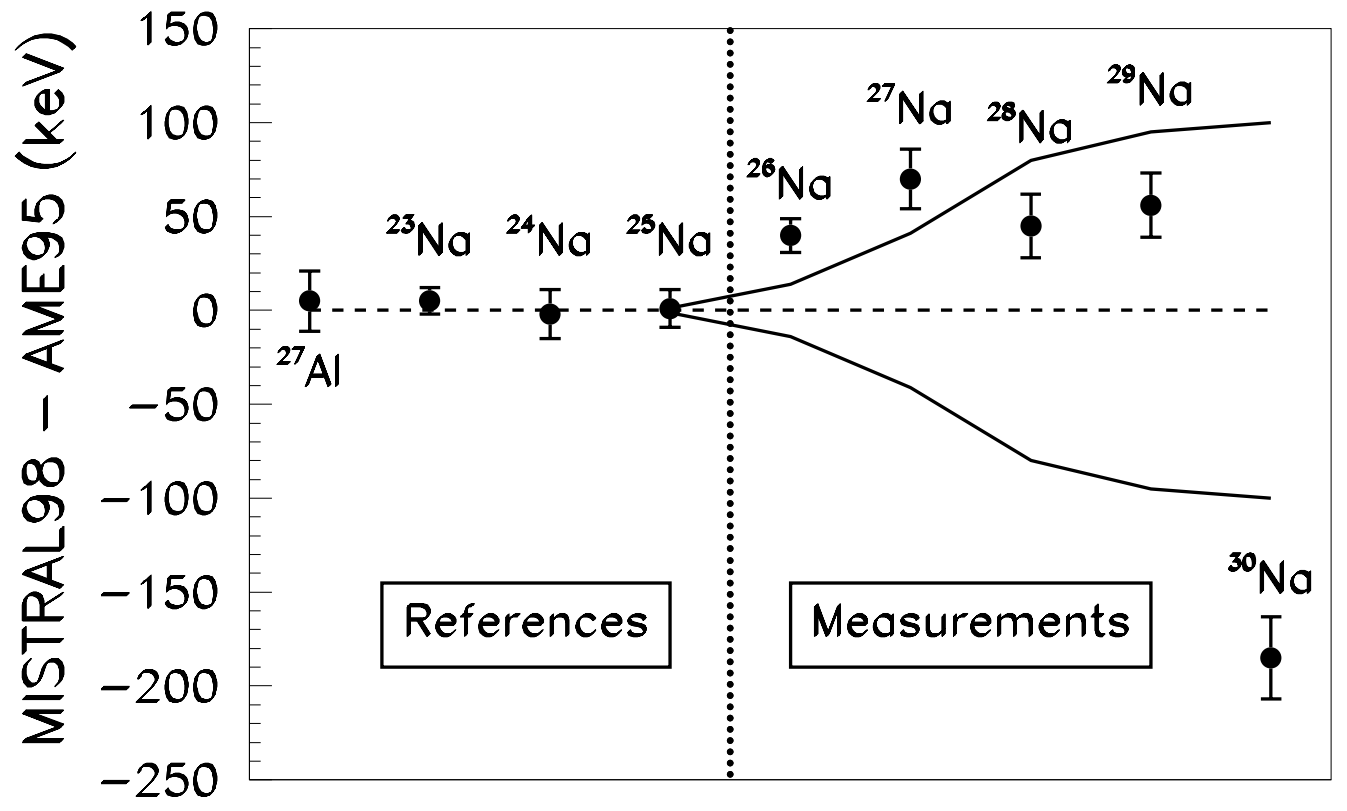

Fig. 1. The Mistral results compared to the AmE'95 for ${ }^{27} \mathrm{Al}$ and for the sodium isotopes from $A=23$ to 30 . The zero line represents the values from the mass table and the two continuous symmetrical lines represent the table uncertainties.

at ISOLDE / CERN during the summer 1997. This spectrometer uses a technique of radiofrequency excitation of ion kinetic energy in a homogeneous magnetic field. The cyclotron frequency is determined with the help of the radiofrequency field applied at the beginning and at the end of a cyclotron orbit. Ions may only reach the final detector if this radiofrequency $f_{R F}$ is related to their cyclotron frequency $f_{c}$ when $f_{R F}=\left(n+\frac{1}{2}\right) f_{c}$ which leads to a "zero" net effect of the two modulations (1),(2),(3). The mass is obtained by the comparison of the cyclotron frequency of the unknown mass to that of a reference mass. The product of their mass by their cyclotron frequency is constant at a given field strength.

During three data taking periods, we measured masses of short lived isotopes of $\mathrm{Mg}, \mathrm{Na}, \mathrm{Ne}, \mathrm{Al}, \mathrm{Ti}, \mathrm{Ca}$ and $\mathrm{K}$. These measurements extend from the valley of stability to ${ }^{30} \mathrm{Na},{ }^{26} \mathrm{Ne}$ and ${ }^{32} \mathrm{Mg}$. Eight of them have a half-life under 1s, the shortest half-life being $31 \mathrm{~ms}$ for ${ }^{28} \mathrm{Na}$. The sodium results are compared in Fig. 1 to the accepted mass values from the 1995 "Atomic Mass Evaluation" (AmE'95) (4). The average standard deviation (difference in masses divided by the experimental precision) for the reference masses is extremely good (0.34) showing that the quoted precisions are certainly not overestimated. The masses of ${ }^{23-30} \mathrm{Na}$ were measured during two separate runs yielding very consistent results.

The Mistral masses for ${ }^{26} \mathrm{Na}$ to ${ }^{29} \mathrm{Na}$ are in fair agreement with AmE'95 but more precise, our knowledge for the ${ }^{28,29} \mathrm{Na}$ masses are improved by a 


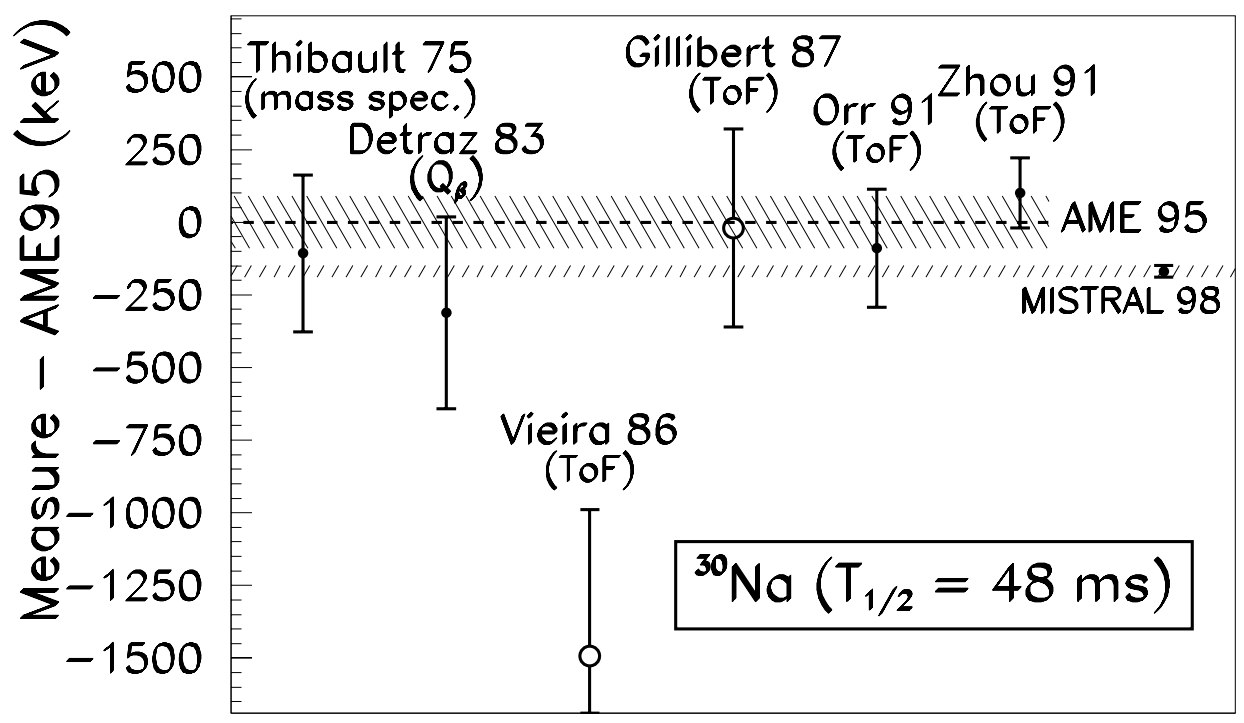

Fig. 2. Comparison of the results of all experiments in which the mass of ${ }^{30} \mathrm{Na}$ has been determined. The dashed area represents the $1 \sigma$ limit of the AmE'95 value, based on the four data represented by full symbols and thick error bars. The measurements represented here were obtained, from left to right, by: mass spectrometry (5) and $\beta$ end-point energy (6) by the Orsay group at CERN; four time-of-flight technique Tof mass determination by two groups at LAMPF (7), GANIL (8), GANIL (10) and LAMPF (9); and by RF mass spectrometry.

factor of five. The mass we derive for ${ }^{30} \mathrm{Na}$ strongly disagrees with the mass table. A closer examination locates the disagreement (see Fig. 2) to only one experiment (9) in which the mass of ${ }^{30} \mathrm{Na}$ was derived from a time-of-flight (TOF) measurement at LAMPF. One can notice in Fig. 2 that this discrepant result superseded an earlier ToF measurement of the same group (7) which is also at strong variance with our result, but in the opposite direction to the recent one. Fig. 2 shows that the MistraL result agrees nicely with all other data, but is at least one order of magnitude more precise.

The resulting values confirm and still slightly enhance the overbinding of $\mathrm{Na}$ isotope at $N=19$ (Fig. 3). Our exploratory experiment on $\mathrm{Mg}$ isotopes shows the same types of results for ${ }^{32} \mathrm{Mg}$ which appears some $200 \mathrm{keV}$ more bound than in AME'95. However because of the poor calibration (11), the measurement of that particular nuclide requires a confirmation. These tendencies for ${ }^{30} \mathrm{Na}(N=19)$ and for ${ }^{32} \mathrm{Mg}(N=20)$ contradict even more $N=20$ being a magic number at $Z=11$ and reinforce the strengh of the deformation starting at $N=19$. This enhancement compared to AME'95 is closer to the HartreeFock calculations of Campi et al. (12) (Fig. 3) where strong deformations were considered. 


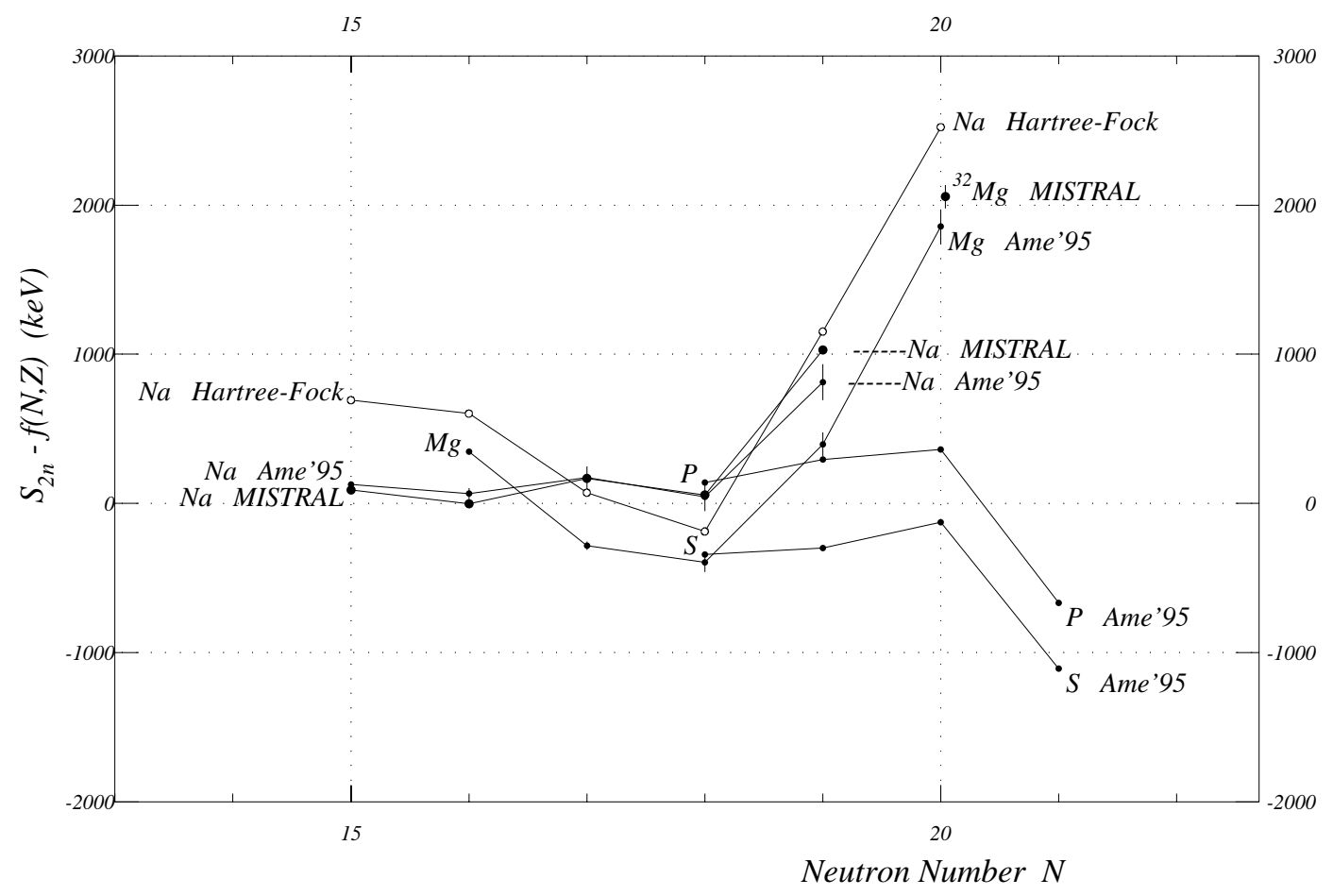

Fig. 3. Two neutron separation energies $\mathrm{S}_{2 n}$ for the very neutron-rich sodium isotopes. A simple function of $N$ and $Z$ has been substracted from $\mathrm{S}_{2 n}$ to show more clearly its fine structure: $f=-3300 \times N+100 \times N \times Z+700 \times Z+39800$. The lines corresponding to Phosphorus (P) and Sulfur (S) show the tendency observed for higher $Z \mathrm{~S}_{2 n}$ curves where $N=20$ is considered a magic number. The strong increase at $N=19$ in the Hartree-Fock predictions is clearly closer to the MisTRAL result than it was to the AME'95 table.

\section{References}

[1] L.G. Smith, Proc. 3rd Int. Conf. Atomic Masses, R. Barber, ed. (University of Manitoba Press, Canada, 1967) 811

[2] D. Lunney et al., Hyp. Int. 99 (1996) 105

[3] C. Toader, Doctoral Thesis, Université de Paris Sud, 1999

[4] G. Audi and A.H. Wapstra,Nucl. Phys. A 595 (1995) 409.

[7] D.J. Vieira et al.,Phys. Rev. Lett. 57 (1986) 3253.

[5] C. Thibault et al.,Phys. Rev. 12 (1975) 644.

[6] C. Détraz et al.,Nucl. Phys. A 394 (1983) 378.

[8] A. Gillibert et al.,Phys. Lett. B 192 (1987) 39.

[9] X.G. Zhou et al.,Phys. Lett. B 260 (1991) 285.

[10] N.A. Orr et al.,Phys. Lett. B 258 (1991) 29 and erratum Phys. Lett. B 271 (1991) 468.

[11] C. Monsanglant, Doctoral Thesis, Unpublished, 2000

[12] X. Campi, H. Flocard, A.K. Kerman and S. Koonin Nucl. Phys. A 251 (1975) 193. 